\title{
Flame-Retardant Bilayer Separator with Multifaceted van der Waals Interaction for Lithium-Ion Batteries
}

Guangfeng Zeng ${ }^{\dagger, \perp}$, Junying Zhao ${ }^{\dagger, \perp}$, Chao Feng ${ }^{\dagger, \perp}$, Dongjiang Chen ${ }^{\dagger}$, Yan Meng ${ }^{\star}$, Bismark Boateng ${ }^{\dagger, \S}$, Ning $\mathrm{Lu}^{\prime}$, Weidong $\mathrm{He}^{*}, \dagger, \S$

†School of Physics, University of Electronic Science and Technology of China, Chengdu 610054, PR China.

†College of Chemical Engineering, Sichuan University, Chengdu 610065, PR China.

${ }^{\S}$ State Key Laboratory of Electronic Thin Film and Integrated Devices, University of Electronic

Science and Technology of China, Chengdu 610054, PR China.

"Department of Breast Cancer Medical Oncology, Tianjin Medical University Cancer Institute and Hospital, Tianjin Medical University, Ministry of Education, Tianjin 300060, PR China. 

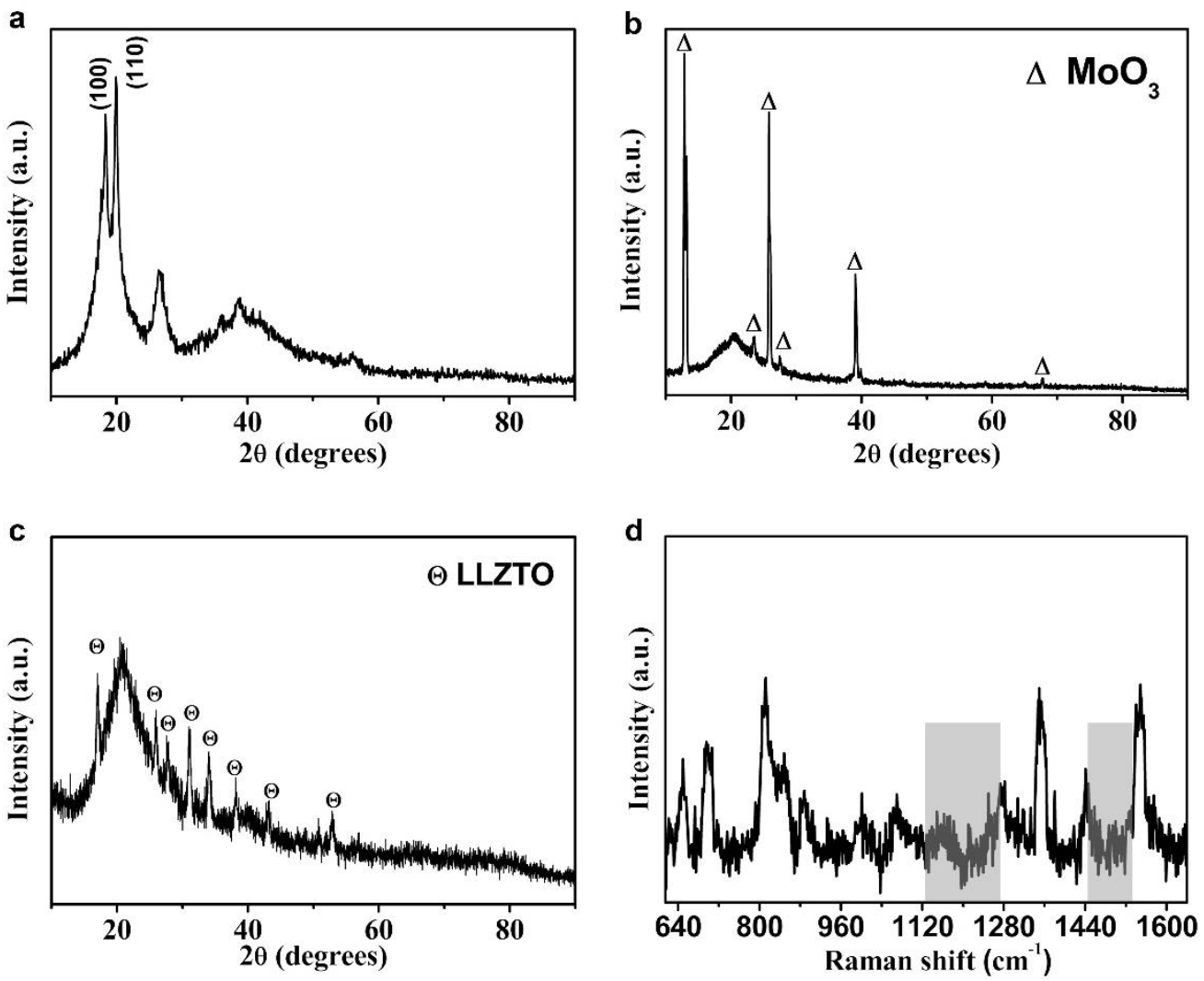

Figure S1 XRD patterns of (a) PVDF-HFP powders, (b) $\mathrm{MoO}_{3}-$ PVDF-HFP separator and (c) LLZTO-PVDF-HFP separator, respectively. (d) Raman spectra of PVDF-HFP separator between the band regions of $615-1640 \mathrm{~cm}^{-1}$. There are no peaks attributed to the $\mathrm{C}=\mathrm{C}$ bond at the band of $1137 \mathrm{~cm}^{-1}$ and $1525 \mathrm{~cm}^{-1}$. 

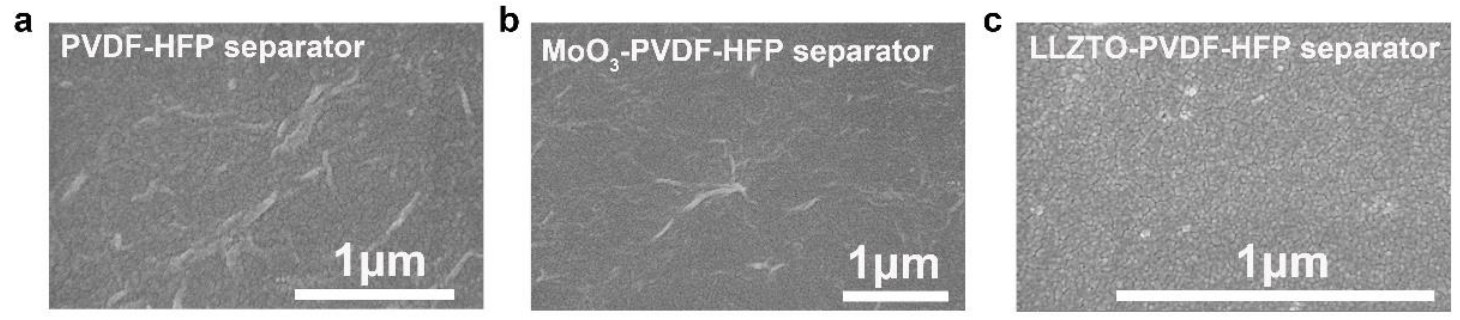

Figure S2 Top-view SEM images of (a) PVDF-HFP, (b) $\mathrm{MoO}_{3}-\mathrm{PVDF}-\mathrm{HFP}$ and (c) LLZTO-PVDF-HFP separators, respectively.

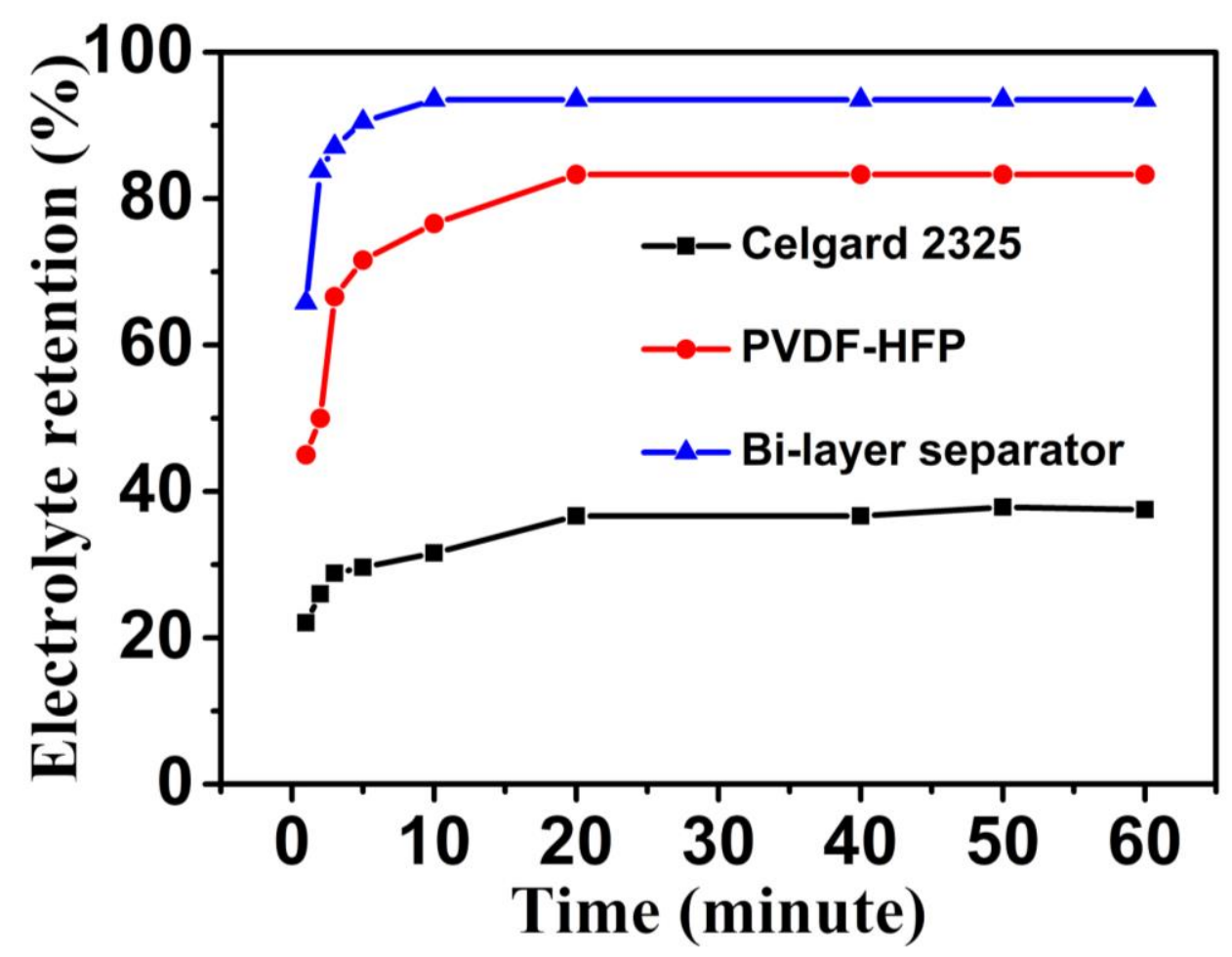

Figure S3 Electrolyte retentions of Celgard 2325, PVDF-HFP and bilayer separators after soaking in liquid electrolyte for different time durations. 

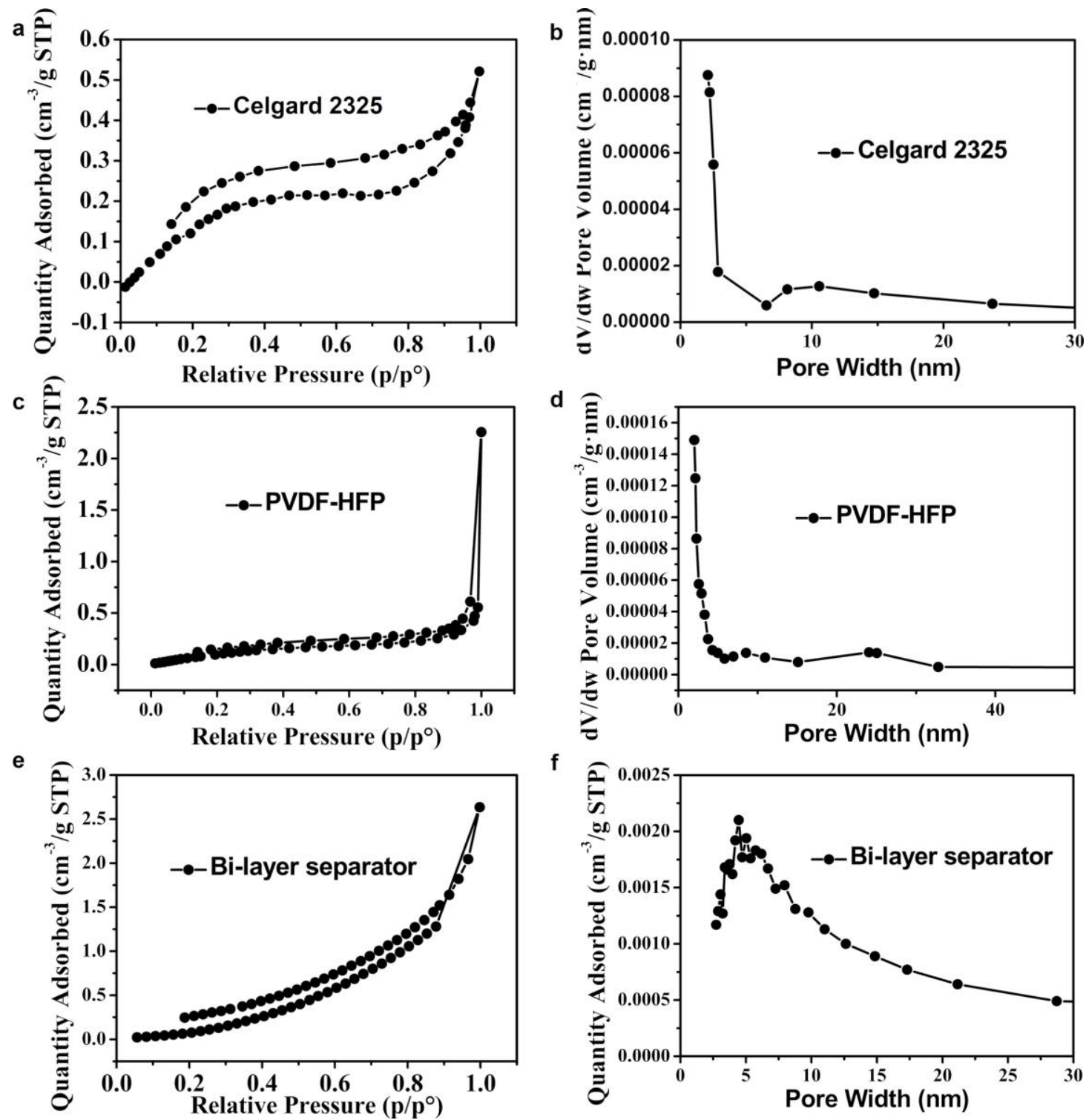

Figure S4 Nitrogen adsorption and desorption isotherms and pore size distributions of (a-b)

Celgard 2325, (c-d) PVDF-HFP and (e-f) bilayer separators, respectively. 
a

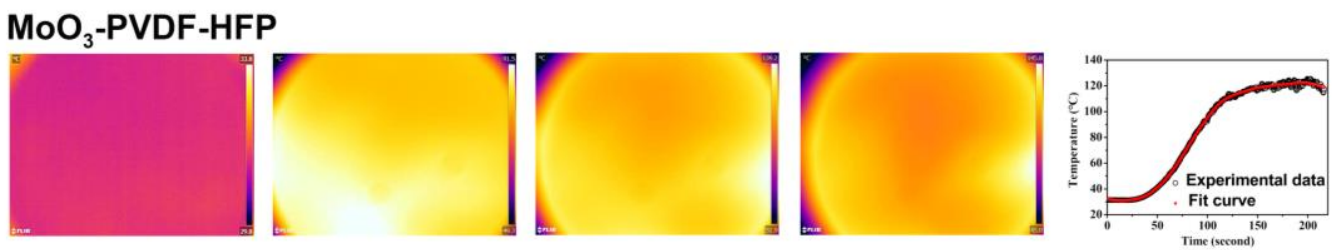

b

LLZTO-PVDF-HFP

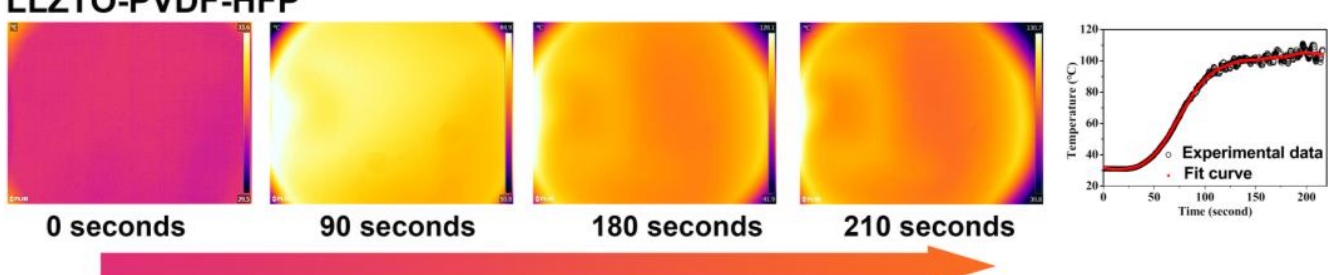

$\mathrm{MoO}_{3}$-PVDF-HFP

Heating

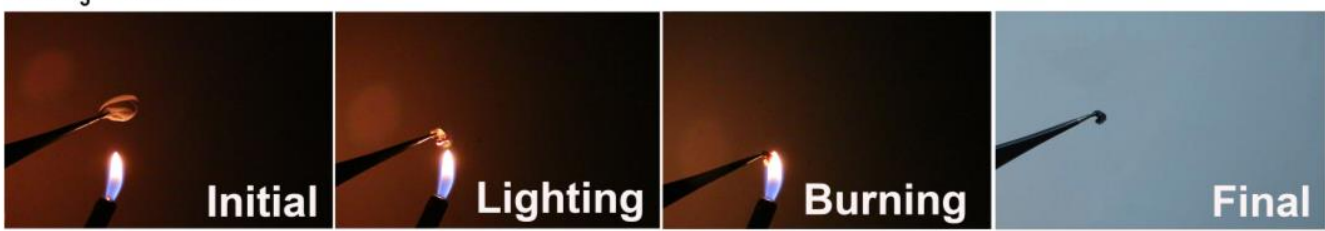

LLZTO-PVDF-HFP

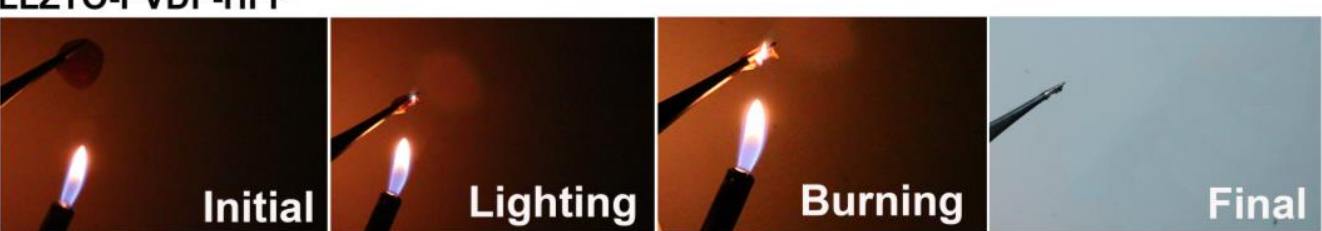

Figure S5 FLIR thermal distributed images (left) and the corresponding time-resolved temperature curves (right) of (a) $\mathrm{MoO}_{3}$-PVDF-HFP separator and (b) LLZTO-PVDF-HFP separator. Digital photographs of combustion test of (c) $\mathrm{MoO}_{3}-$ PVDF-HFP separator and (d) LLZTO-PVDF-HFP separator. 

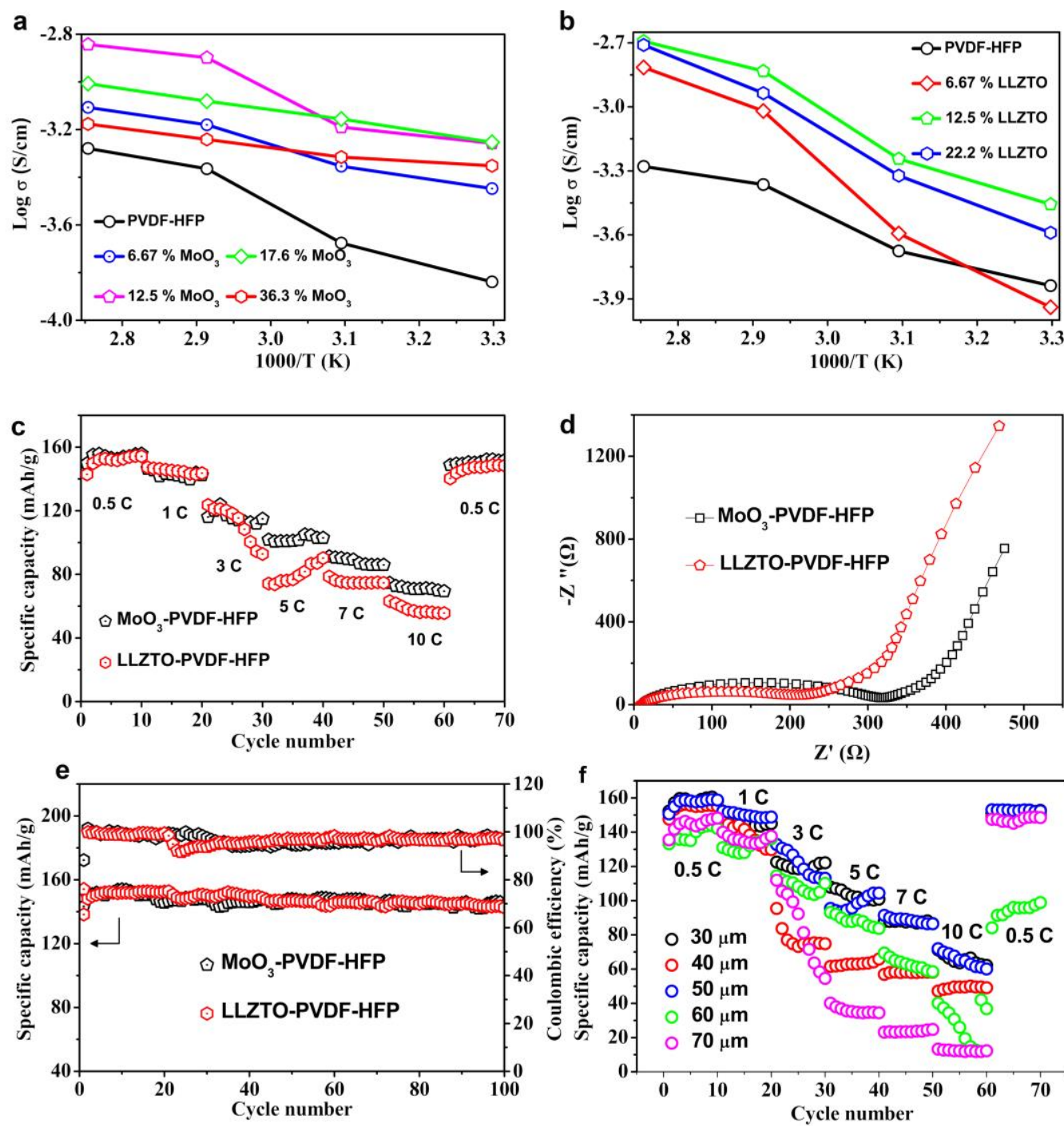

Figure S6 (a) Ion conductivities of $\mathrm{MoO}_{3}-$ PVDF-HFP separators with different contents of $\mathrm{MoO}_{3}(6.67 \%, 12.5 \%, 17.6 \%$ and $36.3 \%)$. (b) Ion conductivities of LLZTO-PVDF-HFP separators with different contents of LLZTO $(6.67 \%, 12.5 \%$ and $22.2 \%)$. (c) Rate performances of Li/LFP cells with $\mathrm{MoO}_{3}-\mathrm{PVDF}-\mathrm{HFP}$ and LLZTO-PVDF-HFP separators. (d) EIS of Li/LFP cells with $\mathrm{MoO}_{3}-$ PVDF-HFP and LLZTO-PVDF-HFP separators. (e) Cyclic performances of Li/LFP cells with $\mathrm{MoO}_{3}-$ PVDF-HFP and LLZTO-PVDF-HFP separators. (f) Rate performances of Li/LFP cells assembled with bilayer separators with different thicknesses. 


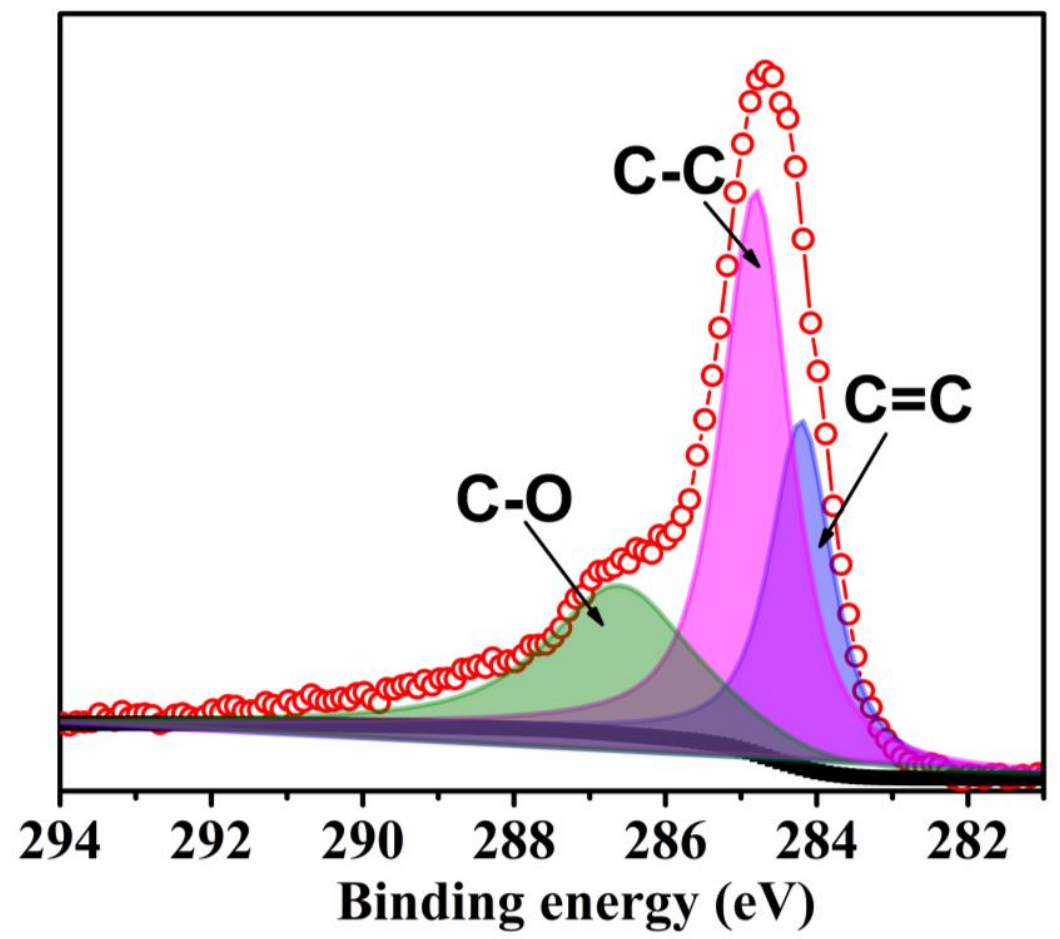

Figure S7 The C 1s XPS spectra of carbon residues of bilayer separator after burning. 


\section{Table S1}

\begin{tabular}{llll}
\hline Separators & Electrolyte uptake & MucMullin number & Thermal shrinkage \\
\hline Celgard 2325 & $91.8 \%$ & 19.2 & $>90 \%$ \\
PVDF-HFP & $104.5 \%$ & 55.2 & $42.1 \%$ \\
MoO $_{3}$-PVDF-HFP & $223.6 \%$ & 14.5 & $10.5 \%$ \\
LLZTO-PVDF-HFP & $442.3 \%$ & 22.9 & $13.1 \%$ \\
Bilayer separator & $372.6 \%$ & 12.6 & $5.3 \%$ \\
\hline
\end{tabular}


Table S2

\begin{tabular}{|c|c|c|c|}
\hline Separators & $\begin{array}{l}\text { Specific } \\
\text { surface area }\end{array}$ & $\begin{array}{l}\text { Average Pore } \\
\text { size }\end{array}$ & Porosity \\
\hline Celgard 2325 & $0.883 \mathrm{~m}^{2} / \mathrm{g}$ & $8.28 \mathrm{~nm}$ & $28.7 \%$ \\
\hline PVDF-HFP & $0.640 \mathrm{~m}^{2} / \mathrm{g}$ & $3.55 \mathrm{~nm}$ & $13.3 \%$ \\
\hline Bilayer separator & $1.492 \mathrm{~m}^{2} / \mathrm{g}$ & $5.18 \mathrm{~nm}$ & $39.8 \%$ \\
\hline
\end{tabular}


Table S3

\begin{tabular}{|c|c|c|c|}
\hline Separators & Uptakes & Discharge capacity (0.5 C) & References \\
\hline $\mathrm{SiO}_{2} /$ Polyetherimide & $188.88 \%$ & $158 \mathrm{mAh} / \mathrm{g}$ & 1 \\
\hline PVDF-HFP / $\mathrm{Al}_{2} \mathrm{O}_{3}$ & $85 \%$ & $107 \mathrm{mAh} / \mathrm{g}$ & 2 \\
\hline $\mathrm{PEI} / \mathrm{SiO}_{2} / \mathrm{PE}$ & $398 \%$ & $141 \mathrm{mAh} / \mathrm{g}$ & 3 \\
\hline $\mathrm{SiO}_{2} / \mathrm{PVDF}$ & $370 \%$ & $160 \mathrm{mAh} / \mathrm{g}$ & 4 \\
\hline $\mathrm{SiO}_{2} / \mathrm{Al}_{2} \mathrm{O}_{3} /$ polyimide & $519 \%$ & $140 \mathrm{mAh} / \mathrm{g}$ & 5 \\
\hline $\mathrm{TiO}_{2}$ doped PVDF-HFP & $330 \%$ & $140 \mathrm{mAh} / \mathrm{g}(0.1 \mathrm{C})$ & 6 \\
\hline Polydopamine / $\mathrm{SiO}_{2} / \mathrm{PE}$ & $80 \%$ & $110 \mathrm{mAh} / \mathrm{g}$ & 7 \\
\hline AlOOH / PVDF & - & $120 \mathrm{mAh} / \mathrm{g}$ & 8 \\
\hline Montmorillonite / PVDF-HFP & $251 \%$ & $91 \mathrm{mAh} / \mathrm{g}$ & 9 \\
\hline Polydopamine / PE & $126 \%$ & $123 \mathrm{mAh} / \mathrm{g}(1 \mathrm{C})$ & 10 \\
\hline $\mathrm{Mg}(\mathrm{OH})_{2} / \mathrm{PE}$ & $115 \%$ & $140 \mathrm{mAh} / \mathrm{g}$ & 11 \\
\hline $\mathrm{Al}_{2} \mathrm{O}_{3} / \mathrm{PVDF}-\mathrm{HFP}$ & $235 \%$ & $100 \mathrm{mAh} / \mathrm{g}$ & 12 \\
\hline Bilayer separator & $372.6 \%$ & $162 \mathrm{mAh} / \mathrm{g}$ & This work \\
\hline
\end{tabular}


Table S4

\begin{tabular}{|c|c|c|c|}
\hline Separators & $\begin{array}{l}\text { Intensity } \\
\text { (G band) }\end{array}$ & $\begin{array}{l}\text { Intensity } \\
\text { (D band) }\end{array}$ & $\mathbf{R}\left(\mathbf{I}_{\mathbf{G}} / \mathbf{I}_{\mathbf{D}}\right)$ \\
\hline PVDF-HFP & 6855 & 8708 & 0.787 \\
\hline $\mathrm{MoO}_{3}-\mathrm{PVDF}-\mathrm{HFP}(6.67 \%)$ & 13315 & 16157 & 0.824 \\
\hline $\mathrm{MoO}_{3}-\mathrm{PVDF}-\mathrm{HFP}(12.5 \%)$ & 11910 & 12540 & 0.950 \\
\hline $\mathrm{MoO}_{3}-\mathrm{PVDF}-\mathrm{HFP}(\mathbf{1 7 . 6 \% )}$ & 10864 & 11681 & 0.930 \\
\hline $\mathrm{MoO}_{3}-\mathrm{PVDF}-\mathrm{HFP}(36.3 \%)$ & 14978 & 16202 & 0.924 \\
\hline LLZTO-PVDF-HFP (6.67 \%) & 9865 & 12035 & 0.820 \\
\hline LLZTO-PVDF-HFP (12.5\%) & 7497 & 7663 & 0.977 \\
\hline LLZTO-PVDF-HFP (22.2\%) & 13753 & 16461 & 0.835 \\
\hline Bilayer separator & 4915 & 4004 & 1.228 \\
\hline
\end{tabular}




\section{References:}

(1) Zhai, Y. Y.; Xiao, K.; Yu, J. Y.; Ding, B. Fabrication of Hierarchical Structured $\mathrm{SiO}_{2} /$ polyetherimidepolyurethane Nanofibrous Separators with High Performance for Lithium Ion Batteries. Electrochimica Acta 2015, 154, 219-226.

(2) Shi, C.; Dai, J. H.; Shen, X.; Peng, L. Q.; Li, C.; Wang, X.; Zhang, P.; Zhao, J. B. High-Temperature Stable Ceramic-coated Separator Prepared with Polyimide Binder $/ \mathrm{Al}_{2} \mathrm{O}_{3}$ Particles for Lithium-ion Batteries. J. Membrane. Sci. 2016, 517, 91-99.

(3) Wang, Z. Y.; Guo, F. L.; Chen, C.; Shi, Y. L.; Yuan, S.; Sun, L. N.; Zhu, J. F. Self-assembly of PEI/SiO2 on Polyethylene Separators for Li-ion Batteries with Enhanced Rate Capability. ACS Appl. Mater. Inter. 2015, 7, 3314-3322.

(4) Yanilmaz, M.; Lu, Y.; Dirican, M.; Fu, K.; Zhang, X. W.; Nanoparticle-on-Nanofiber Hybrid Membrane Separators for Lithium-ion Batteries via Combining Electrospraying and Electrospinning Techniques. 2014, 456, 57-65.

(5) Liang, X. X.; Yang, Y.; Jin, X.; Huang, Z. H.; Kang, F. Y.; The high Performances of $\mathrm{SiO}_{2} / \mathrm{Al}_{2} \mathrm{O}_{3}$-Coated Electrospun Polyimide Fibrous Separator for Lithium-ion Battery. $J$. Membrane. Sci. 2015, 493, 1-7.

(6) Zhang, S.; Cao, J.; Shang, Y. M.; Wang, L.; He, X. M.; Li, J. J.; Zhao, P.; Wang, Y. W. Nanocomposite Polymer Membrane Derived from Nano $\mathrm{TiO}_{2}-\mathrm{PMMA}$ and Glass Fiber Nonwoven: High Thermal Endurance and Cycle Stability in Lithium Ion Battery Applications. J. Mater. Chem. A 2015, 3, 17697.

(7) Dai, J. H.; Shi, C.; Li, C.; Shen, X.; Peng, L. Q.; Wu, D. Z.; Zhang, P.; Zhao, J. B. A Rational Design of Separator with Substantially Enhanced Thermal Features for Lithium-ion Batteries by 
The Polydopamine-ceramic Composite Modification of Polyolefin Membranes. Energy Environ. Sci. 2016, 9, 3252-3261.

(8) M. Schäfter, J. H.; Niemöller, A.; Winter, M.; Lex-Balducci, A.; Obeidi, S. Boehmite-based Ceramic Separator for Lithium-ion Batteries. J. Appl. Electrochem 2016, 46, 69-76.

(9) T. Prem Kumar, M. R.; L. Zolin, G. S.; A. Manuel, C.G. Montmorillonite-Based Ceramic Membranes as Novel Lithium-ion Battery Separators. Ionics 2014, 26, 943-948.

(10) Ryou, M. H.; Lee, Y. M.; Park, J. K.; Choi, J. W. Mussel-inspired Polydopamine-Treated Polyethylene Separators for High-power Li-ion Batteries. Adv. Mater. 2011, 23, 3066-3070.

(11) Yeon, D.; Lee, Y. J.; Ryou, M. H.; Lee, Y. M. New Flame-Retardant Composite Separators Based on Metal Hydroxides for Lithium-ion Batteries. Electrochimica Acta 2015, 157, 282-289.

(12) Shen, X.; Li, C.; Shi, C.; Yang, C. C.; Deng, L.; Zhang, W.; Peng, L. Q.; Dai, J. H.; Wu, D. Z.; Zhang, P.; Zhao, J. B. Core-shell Structured Ceramic Nonwoven Separators by Atomic Layer Deposition for Safe Lithium-ion Batteries. Appl. Sur. Sci. 2018, 441, 165-173. 